\title{
Reflexiones acerca de la salud ambiental
}

\section{Reflections on environmental health}

DOI: $10.7203 / D C E S .36 .12990$

\author{
José Carrasquer \\ Universidad de Zaragoza, josecarr@unizar.es
}

Adrián Ponz

Universidad de Zaragoza, adrian.ponz@unizar.es

Beatriz Carrasquer

Universidad de Zaragoza, becarras@unizar.es

María Victoria Álvarez

Universidad de Zaragoza, valvarez@unizar.es

\begin{abstract}
RESUMEN: Los conceptos salud y ambiente son utilizados de forma habitual desde diversos ámbitos sociales y científicos, lo que provoca que su significación sea muy amplia y en muchas de las ocasiones sin unos límites claros. En este trabajo se aportan una serie de planteamientos en los que se fijan esos límites y se concretan los aspectos en los que se considera que su utilización debiera ampliarse o incidir con más énfasis, especialmente en el ámbito educativo, en el que a pesar de los esfuerzos dedicados en los últimos decenios, los resultados no parecen ser los esperados. Se considera que planteamientos centrados en aspectos naturales sin tener en cuenta otros como la sostenibilidad social deben ser reconducidos.
\end{abstract}

Palabras clave: Promoción de la salud, educación para la salud, educación ambiental, ecologismo, participación ciudadana

ABSTRACT: The concepts of health and environment are commonly used in various social and scientific fields. That makes their significance very broad and with unclear limits in many cases. This paper provides diverse approaches in which these limits are set. Those aspects whose use should be extended or those which should be more emphasized have been specified as well. In this respect, the educational field takes importance. In spite of the efforts in recent decades, results do not seem to be as expected. It is considered that approaches focused on natural aspects without taking into account others such as social sustainability should be redirected.

KEYWORDS: Health promotion, health education, environmental education, ecologism, citizen participation

Fecha de recepción: agosto de 2018 Fecha de aceptación: octubre de 2018

Este trabajo forma parte del Proyecto COMSAL_EDU2013-46664-P, dirigido por el profesor Valentín Gavidia. José Carrasquer, Adrián Ponz y María Victoria Álvarez también pertenecen al Instituto Universitario de Investigación de Ciencias Ambientales de Aragón, de la Universidad de Zaragoza y al Grupo Beagle de Investigación de Didáctica de las Ciencias Naturales, financiado por el Gobierno de Aragón y el Fondo Social Europeo. 


\section{INTRODUCCIÓN}

Las consideraciones que se presentan en este trabajo se enmarcan en el entorno (entendido también como ambiente o medio) y la salud y asimismo, se dirigen hacia la educación acerca de los factores ambientales que afectan a las personas en su salud y de cómo el comportamiento de éstas afecta al medio. Los factores ambientales implicados en la salud humana se pueden concretar en aquellos derivados de la contaminación del aire, del agua, del suelo, del consumismo y de las catástrofes naturales o las provocadas por las personas. Por otra parte, la educación en salud ambiental debe tratar de conseguir la adquisición de todas aquellas competencias o habilidades que tengan que ver con la asunción de cómo intervienen la personas en los ambientes, la contribución a su mantenimiento o de su construcción, para alcanzar ambientes más saludables para las personas, como individuos y como colectividades locales o universales (Gavidia, 2016).

El universo en el que la mayoría de los jóvenes se desenvuelven habitualmente, en sus primeros años, se reduce en gran medida a la familia, la escuela, las amistades y a los servicios sanitarios (Arasa et al., 1991; Talavera y Gavidia, 2013; Pueyo, Carrasquer, Álvarez y Ponz, 2016). La escuela, como el espacio destacado en el que se coopera a la construcción de los comportamientos de los individuos, es la que se suele señalar como prioritaria para promocionar la salud "[...] por su importante contribución a la configuración de la persona en todas sus dimensiones.” (González de Haro, 2008, p.125). Pero prioritaria no significa única, dado que para que la estructura educativa formal funcione correctamente ha de estar implicada también la comunidad, en la que se incluyen además de los ciudadanos las estructuras administrativas (Marchioni, 2004, p.113). Cada vez con mayor interés, por su trascendencia, habrá que contemplar una ventana abierta a ese universo, dado que no es simplemente el cercano físicamente y así se pone de manifiesto en la Declaración de Independencia del Ciberespacio de Barlow (1996), sino también el lejano y global que sobre todos actúa.

Sin embargo, en nuestro país, la coordinación entre los servicios educativos, sanitarios, familias y estructuras políticas/administrativas, no ha sido la suficiente (Vélaz de Medrano, Manzano y Blanco, 2013, p.120), dado que la independencia de cada uno de ellos y la falta de concreción explícita de sus responsabilidades (Casado, 2008; García Herrero, 2017) ha podido impedir una mayor rentabilidad en la formación deseada, lo que ha ocasionado la aparición de nuevas normativas o legislaciones que faciliten esta coordinación (Conselleria de Educación, Investigación, Cultura y Deporte, 2017). Por otra parte, con el paso de los años la importancia del medio social en el que desenvuelven los jóvenes está teniendo una relevancia fundamental.

El ecologismo ${ }^{1}$ políticamente correcto, ecologismo superficial, se suele referir habitualmente a la contaminación, al agotamiento de los recursos o al reciclaje y la salud de las personas que viven en zonas desarrolladas; es decir se ve el planeta desde una perspectiva de lo que puede afectar a las condiciones de vida del mundo desarrollado, no importando demasiado el exceso de explotación del medio.

La iniciativa SCALE (Comisión de las Comunidades Europeas, 2003), de la Comunidad Económica Europea sobre Medio Ambiente y Salud pretende dar protección a los colectivos con mayor riesgo de vulnerabilidad, considerando con una mayor relevancia al ambiente como un factor determinante de la salud. Inicialmente SCALE, al igual que ocurre con otras normativas que generan proyectos ambientalistas podría parecer, y tal vez lo es, que las directrices se fundamentan en la aceptación de la importancia del buen estado de los ecosistemas, así como su vulnerabilidad; sin embargo, y tal como ocurre con otras normativas, por ejemplo, en la Estrategia Europea para la Protección del Medio Marino, su desarrollo y actuaciones se centran en las vertientes económicas, pérdida de creación de riqueza y de puestos de trabajo (De Marcos, 2011), ha centrado sus

\footnotetext{
${ }^{1}$ En este trabajo, independientemente de que en las citas textuales de otros autores/as se utilice el término ecología para referirse al ecologismo, en el resto del texto se mantendrá en la redacción la diferencia entre ecología y ecologismo, propia del castellano.
} 
esfuerzos en legislar acerca de aquellos procesos industriales o económicos que generan efectos negativos de forma sustancialmente relevante en la salud de las personas, situando en segundo término al medio: "El fomento de la salud [de las personas] ha sido siempre uno de los motores de la política de protección del medio ambiente” (Comisión de las Comunidades Europeas, 2003, p.6).

Sin embargo, existe otro planteamiento del ecologismo, el profundo, para el que el ser humano, como especie, no puede separarse del todo pues forma parte de él, formulación que sitúa en el centro del pensamiento a la naturaleza y no a las personas (Naess, 1973, p.95; Sotolongo y Delgado, 2006, p.169).

El presente texto es consecuencia de las discusiones acerca de la salud ambiental y de cómo se ha venido trabajando en las aulas, así como el interés y las limitaciones de diversos recursos utilizados en ellas y que tienen como consecuencia la construcción de determinados contenidos actitudinales.

Estos pensamientos y preguntas no se quedan en cuestiones amplias y generales, sino que también preocupan aspectos como: ¿Tendrán estos resultados educativos relación con el número de accidentes laborales, agresiones sexuales a mujeres o delitos contra la salud de las personas o el medio? Estas preguntas y otras similares deben ser planteadas en una educación que trata de formar ciudadanos responsables, críticos y solidarios (Imagen 1).

IMAGEN 1. Contenidos de salud ambiental.

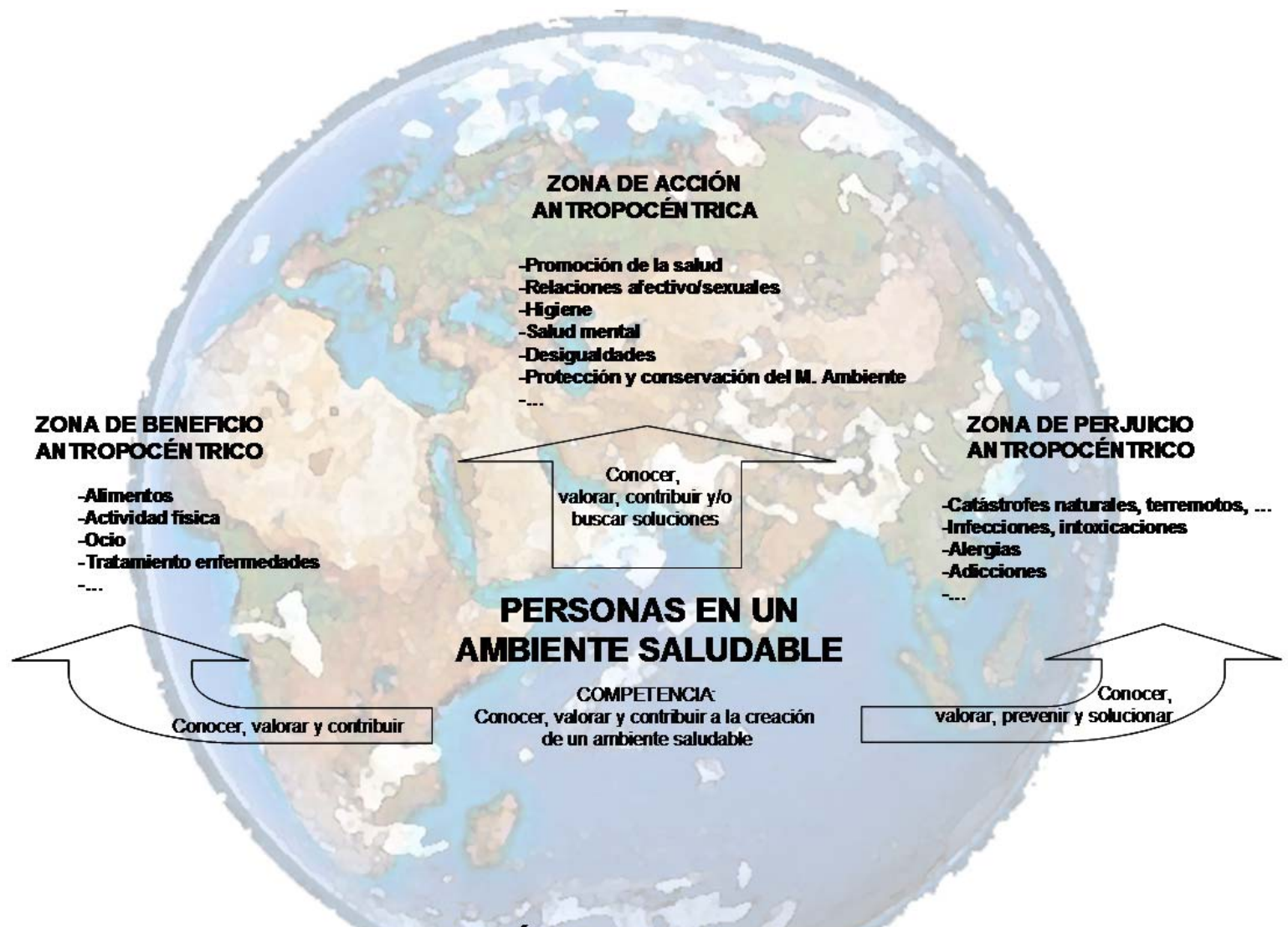

FORMACIÓN EN SALUD AMBIENTAL

Fuente: elaboración propia 


\section{EL PROBLEMA DE CÓMO PLANTEAR EN EL AULA LA SALUD AMBIENTAL}

Los planteamientos holísticos, debido a la amplitud del significado de los conceptos ambiente, educación ambiental, salud, educación para la salud, promoción de la salud, etc., puede conducir a manejar tal cantidad de variables, contenidos y perspectivas que provoquen planteamientos difíciles de llevar a cabo, pero tal como indica Zabala (1999) “[...] no debería extrañarnos que enseñar fuese difícil” (p.193).

El estudio del ambiente, el ecosistema como cosmovisión (García Díaz, 2003), en el que se incluyen los conocimientos ecológicos (estudio de las relaciones entre los componentes de los ecosistemas desde metodologías científicas), pero también los conocimientos necesarios para el planteamiento denominado inicialmente ecologista (interacción de los conocimientos ecológicos con los filosóficos, políticos, sociales, etc.), puede resultar complejo para el trabajo en las aulas de la mayoría de los niveles educativos no universitarios y sin duda también para la mayoría de la sociedad, incluido parte del profesorado. Estas propuestas enmarcadas en las teorías de la complejidad, enfrentadas con la cultura de la superficialidad, nos sugieren una ecología global, un planeta eco-socio-sistémico, un multiperspectivismo, que conduce a un conocimiento escolar deseable (García Díaz, 2003, p.87-88), difícil de generalizar en los niveles de enseñanza obligatorios, incluso teniendo en cuenta su aplicación de forma progresiva, mediante hipótesis de progresión (García Díaz, 2004), aumentando la interdisciplinariedad y la complejidad, desde los primeros años de escolarización. Los puntos de arranque son los nudos principales, a partir de los cuales se forman las tramas de contenidos construyendo un "cuore” o núcleo central de aprendizaje. Deseo loable e impecablemente estructurado desde el pensamiento teórico, pero el tiempo demuestra su dificultad, lo que obliga a seguir realizando esfuerzos en el aprendizaje y la formación del profesorado para poder llevar a cabo una mejor aplicación en las aulas.

García Díaz (2004, p.37) describe los nudos a partir de los cuales crear las tramas de contenidos, redes de problemas socioambientales transdisciplinares relevantes alrededor de los cuales organizar el conocimiento progresivamente. Un ejemplo citado por el autor para acercarse a la complejidad de cualquier acción social o económica o política es el siguiente:

"[...] guerras que se comienzan y que siguen un curso no previsto; productos que se comercializan y que provocan indeseables consecuencias para la salud y para el propio mercado; contaminaciones puntuales que determinan efectos globales como el cambio de clima o el deterioro de la capa de ozono; [...]” (García Díaz, 2004, p.45).

Con un planteamiento similar pero partiendo de formulaciones menos ambiciosas, Harlen (2010) define el concepto de grandes ideas de las ciencias alrededor de las que trabajar los contenidos y concreta algunas, entre las cuales llama la atención sobre aquellas que permiten tomar decisiones que afectan: 1) a la salud y el bienestar de los demás, 2) al ambiente y 3) al uso de energía.

Lo dicho respecto a la educación ambiental puede plantearse asimismo respecto a la salud. La idea de salud en sí misma es un sistema complejo del que forman parte las personas y el medio en el que viven (Ponz, Carrasquer, Alvarez y Carrasquer, 2017).

Sin embargo, a pesar de estos enfoques deseables, los efectos de la Educación para la Salud y de la Educación Ambiental no parecen haber dado resultado con las actuaciones llevadas a cabo en los últimos treinta años, ¿tal vez es poco tiempo? o estos métodos de enseñanza no han llegado a las aulas de forma amplia, por diversos motivos, y por tanto tampoco se han hecho visibles los posibles y deseables resultados positivos. 


\section{EL MARCO TEÓRICO PARA LA SALUD AMBIENTAL}

En ocasiones, los métodos de estudio para realizar las investigaciones de los expertos, se adaptan, se configuran, teniendo en consideración la realidad del mundo complejo, de sus interrelaciones y variables. Asimismo, en ocasiones, esos mismos métodos de estudio se quieren utilizar como planteamientos didácticos funcionales; es decir, los mismos que se consideran óptimos para el estudio de expertos, se quieren utilizar para la educación de jóvenes inexpertos. Esto conduce a los enfoques globales, utilizados por ejemplo en la Educación Infantil y a los holísticos en cualquier otro nivel.

Si se asume que la enseñanza tiene una indiscutible función social, los ciudadanos han de comprender la realidad que les rodea y ser capaces de querer y poder intervenir en ella, lo que implica necesariamente una formación compleja. Entonces, ¿por qué se instauran las disciplinas? Las asignaturas nacen con la dificultad de los contenidos a manejar, pero no son el fin de la enseñanza, sino una herramienta para comprender la complejidad del medio y actuar en él (Zabala, 1999).

\subsection{Respuestas a preguntas holísticas, complejas}

¿Es posible construir todos los contenidos necesarios para responder una pregunta, en el propio marco de una investigación?, o bien ¿es preciso tener adquiridos conocimientos previos para no perdernos en una maraña de aprendizajes cruzados, con riesgo de perdernos, antes de alcanzar una solución?

Desde el marco de las teorías implícitas (Rodrigo, 1994) se incide en que los aprendizajes interdisciplinares (y también se debería añadir interpersonales) han de basarse en la construcción previa de esquemas de conocimiento disciplinares, para poder ser utilizados y relacionados en el contexto en el que se han construido, pero también en contextos diferentes. Se puede decir que el constructo de teorías implícitas señalado por Rodrigo, para niños/as y adultos, se refiere a un conjunto de afirmaciones de conocimiento relacionadas mediante algún concepto, que se enmarcan en un dominio determinado (p.e. la salud ambiental) y que generan algo semejante a una teoría científica, pero sin alcanzar una organización y coherencia que permita entenderla como tal. Por otra parte, la persona poseedora de esos conocimientos no es consciente (de ahí que se denomine implícita) de que es capaz de utilizarlos para dar respuesta a preguntas o acontecimientos del mundo físico o social, construyendo su realidad. La autora indica la analogía de unas gafas que obligan a ver la realidad de una forma determinada, pero sin ser consciente de que las gafas existen.

Muy a menudo estas teorías implícitas se construyen en procesos de aprendizaje colectivos mediante la realización de actividades conjuntas, mediante negociaciones o discusiones de ámbito cultural, lo que puede ocasionar significaciones compartidas, teorías implícitas, tramas de conocimientos comunes en grupos de personas que pueden intercambiar opiniones entre ellas. Los dominios de conocimientos generados alrededor de problemas o situaciones se enriquecerán con creencias conducentes a actitudes en el comportamiento.

En un momento determinado la persona necesitará posiblemente de los contenidos para debatir o discutir acerca de asuntos del dominio concreto, sin embargo cuando necesite tomar decisiones acerca de su comportamiento usará aquellas creencias del dominio, pero en función del contexto donde deba emplearlas, por lo que tan importante como la adquisición de conocimientos y creencias será la habilidad del sujeto para elegir cuáles de sus conocimientos son los más adecuados para dar respuesta en un contexto concreto (Pozo, 1993, p.198-200). De lo anterior se puede concluir que la generación de conocimiento colectivo es fundamental para generar comportamientos determinados.

Sin duda las aulas, aunque la procedencia y formación de los estudiantes no sea homogénea, facilitan el lugar y tiempo de cooperación y negociación, mediante las cuales se garantiza el espacio 
participativo y los intercambios de conocimiento, no obstante es necesario tener presente que las creencias que se configuran como la identidad social de un grupo, habitualmente se considera que no deben ser discutidas, analizadas o verificadas, al contrario de lo que se haría si fuesen construcciones científicas (Castorina, Barreiro y Toscano, 2005).

Por lo anteriormente expuesto se deben entender las representaciones sociales como un punto de partida para trabajar sobre la salud ambiental, dado que esas representaciones que se han construido en un entorno social y con aportaciones personales son la base para el trabajo educativo (Meira, 2013).

Para Pareja (2011) no siempre es posible abarcar todos los contenidos necesarios para aprender a partir de una posible pregunta que conduce a una investigación; no es posible globalizarlo todo. Tal vez lo más oportuno es plantearse qué contenidos son los mínimos imprescindibles que, previamente, ha de tener el aprendiz para adquirir un aprendizaje funcional, globalizado.

Por otro lado, hay que tener presente la diversidad cognitiva del grupo, dado que los grupos de clase no tienen por que tener conjunción de ideas, sino que suelen ser agrupaciones artificiales sin intereses coincidentes ni conocimientos homogéneos. Habitualmente se quiere, por una parte, que la enseñanza sea inclusiva, para lo cual hay que minimizar las competencias a alcanzar, pero por otro lado se olvida que este planteamiento, y aún a pesar de él, sigue dejando un porcentaje excesivo de jóvenes fuera del sistema educativo. $\mathrm{Y}$ aunque un porcentaje importante de los estudiantes siguen adelante, lo hacen con un bagaje de aprendizaje bastante reducido y aún así, también diverso (¿inclusividad?).

¿Con qué parte de los estudiantes y con qué posibilidad de éxito se quiere trabajar de manera holística? La velocidad y calidad de construcción de conocimientos en un aula será diferente, sin embargo, el tratamiento de los contenidos seguirá adelante para todos/as igual. Se observa que a pesar de los esfuerzos económicos y pedagógicos no se llega a alcanzar solución a esta situación (OECD, 2017).

Si bien esta reflexión anterior va dirigida a los estudios no obligatorios es preciso considerar que esta situación, de forma general, es consecuencia de un fracaso paulatino del Sistema en su formación (Fernández Enguita, 2016, p.30), por lo que, en las etapas intermedias, hay que considerar que, su formación habrá tenido deficiencias que se habrán manifestado también en los niveles anteriores.

Los contenidos cognitivos propios del curriculum se han de integrar con los elementos de socialización como, por ejemplo, la salud y el respeto al medio, entre otros, pero estos elementos se suelen presentar como secundarios respecto a los primeros (Marchioni, 2004, p.123), cuando debieran ser los más importantes, si se considera que la finalidad del sistema educativo es el desarrollo pleno de las personas y que éste permita su integración en el sistema social.

\subsection{La socialización de preguntas de ciencias}

Marchioni (2004, p.129-130) es crítico con los avances en la formación social y lo fundamenta en que existe preocupación por la sostenibilidad ambiental, pero no se hace nada por la sostenibilidad social; no todo el mundo tiene la posibilidad de participar en igualdad de condiciones; no es posible la sostenibilidad ambiental, sin una sostenibilidad social. Los propios estudios acerca del fracaso del Sistema Educativo, puesto de manifiesto por el porcentaje de jóvenes que lo abandonan tempranamente, ratifican, además del fracaso del propio sistema, la diferencia notable de éste, entre sexos o de unos lugares a otros de un mismo país. Si se pone como ejemplo España, la media de abandono educativo temprano (ciudadanos/as entre 18 y 24 años que no han finalizado sus estudios obligatorios y no siguen ningún otro tipo de formación) se sitúa en el 19,0\%; el de las mujeres es un $15,1 \%$ y el de los hombres un $22,7 \%$. Si se comparan los datos por regiones 
todavía es más exagerada la diferencia entre el 7,9 de abandono de estudiantes en el País Vasco comparado con el 26,8 \% de las Islas Baleares (Ministerio de Educación, Cultura y Deporte, 2017).

El fin último de las intenciones educativas parecen ser aceptadas por los expertos, los contenidos y las competencias pretendidas también, pero las metodologías no, o al menos no son las que se están llevando a cabo de forma real o no son las planteadas como más eficaces.

Algunos autores/as critican abiertamente la pedagogía centrada en competencias siempre y cuando la experiencia que dirige o indica las competencias a alcanzar provenga de un planteamiento que ha fracasado socialmente. Estas críticas se fundamentan según Bula (2011, p.34) en que "nuestros saberes, costumbres, valores y maneras de obrar", no han sabido dar respuesta a las crisis ambientales, y el sistema educativo basado en competencias, fija éstas de antemano y por lo tanto la continuación de la falta de éxito está servida. Para este autor la educación ambiental ha de ser crítica frente a la educación por transmisión y se pregunta qué competencias se pueden fijar para que alcancen los ciudadanos cuando se está fracasando frente a las crisis ambientales conocidas.

Los problemas ambientales y la repercusión en la salud preocupan a los ciudadanos que no perciben mejoras. Esta percepción negativa, lejos de mejorar y a pesar de los esfuerzos de determinados colectivos sociales y políticos, va en aumento debido a una actualidad nefastamente permanente de conflictos, guerras, migraciones, causados 0 acrecentados por problemas ambientales como la sequía o la falta de alimentos en determinados lugares del mundo. Y esta sensación de indefensión ante catástrofes naturales o no, epidemias, guerras, no es solamente una impresión de los ciudadanos: las presiones económicas, políticas, someten al medio a situaciones irreversibles a medio plazo, lo que ocasiona "repercusiones no sólo en el ámbito natural, sino también personal, social y económico.” (Álvaro, Mayoral y Gavidia, 2017, p.811).

Por otro lado, el concepto de salud está en continua construcción en un mayor número de ciudadanos, pero cuando se vincula con el concepto ambiente la complejidad del asunto tratado aumenta exponencialmente y puede llegar a ser inabarcable. Por ejemplo, cuando Tobón (2007, p.17) en su definición de competencia introduce "la meta de contribuir al desarrollo personal, la construcción y afianzamiento del tejido social, la búsqueda continua del desarrollo económicoempresarial sostenible y el cuidado y protección del ambiente y de las especies vivas", cualquier aprendizaje puede conducir a alcanzar algún aspecto objetivo que se pudiera incluir en ella (Bula, 2011, p.36). Se puede considerar como una competencia vacía por exceso de amplitud.

La competencia para opinar, proponer o resolver situaciones de salud ambiental, así como de cualquier otro tipo necesita de experiencias y entrenamiento. El aprendiz necesita de instrucciones, secuencias y algoritmos escritos, el experto no, éste ya tiene las habilidades adquiridas y desarrolladas en un ámbito determinado, la habilidad se adquiere mediante experiencias en las que sí hubo guías iniciales o propuestas de actuación (Bula, 2010, p.3). La habilidad inicial del aprendiz poco desarrollada y dirigida por programas guías o recetas (Furió y Gil Pérez, 1978) será paulatinamente reemplazada en el experto por "discriminaciones situacionales acompañadas de respuestas asociadas” (Dreyfus y Dreyfus, 1980; Dreyfus \& Dreyfus, 2003, p.108), pero siempre con capacidad de alteración o modificación de respuesta asociada en función de las nuevas situaciones.

Jean Henri Fabre en su libro acerca de las curiosidades de la vida de los insectos (1882, p.166) afirma que, "[...] el insecto sabe cómo hacer frente a lo accidental siempre que el nuevo acto no salga del orden de las cosas que lo ocupa en este momento." ${ }^{2}$. Es decir Fabre considera que, p.e., una avispa albañil puede solucionar problemas respecto a un asunto concreto, la construcción y reparación de su nido de barro cuantas veces sea preciso, mientras que el acto no haya terminado; pero si el nido es dañado cuando el insecto ha pasado a un acto posterior, p.e., introducir presas en el interior del nido, éstas se caerán y no sabrá responder a la nueva situación; terminará depositando el huevo y cerrando un nido vacío.

\footnotetext{
2 “[... l'insecte sait faire face à l'accidentel pourvu que le nouvel acte ne sorte pas de l'ordre de choses qui
} l'occupe en ce moment." 
Mauricio Maeterlinck apoyándose en esta idea de Fabre y refiriéndose a la siempre debatida inteligencia animal dice que cuanto más restringida sea la "situación” y haya asociada una respuesta adecuada, mayor será la inteligencia en contraposición al instinto: “ [...] La sola diferencia [entre la inteligencia y el instinto] consiste en que la una puede detenerse, replegarse sobre sí misma, hacerse consciente, darse cuenta del punto donde se encuentra [la inteligencia], mientras que la otra marcha recta y ciegamente adelante [el instinto].” (Maeterlinck, 1967, p.130).

La utilización del concepto intuitivo, por Dreyfus (2004) cuando se refiere a una decisión rápida y nada analítica ni meditada de un experto debida a sus experiencias propias, dista de la utilización por Fabre o Maeterlinck que se refieren a un comportamiento debido a un instinto in nato.

El concepto intuitivo, entonces, podría vincularse con el concepto de entrenamiento, entendido en la especie humana como la capacidad de "[...] fortalecer, desarrollar o perfeccionar ciertas competencias con vistas a un fin.” (Marina, 2012, p.88). Para este autor los animales, al no conocer el fin con el que realizan determinadas actividades de aprendizaje, simplemente llevarían a cabo actividades de ejercitación, pero que igualmente facilitarían la respuesta adecuada ante situaciones concretas, de forma similar a cuando actúan mediante el instinto in nato.

\section{LA PARTICIPACIÓN CIUDADANA COMO FUENTE DE RESPUESTAS}

Las taxonomías hechas por las personas son siempre artificiales e imperfectas, y entre otras funciones cubren la de hacer más fácil la transmisión de conocimientos. Diversos autores dividen a las personas en dos colectivos, el cuerpo político y la ciudadanía (Hervás de la Torre, 2011, p.149). El cuerpo político configurado por el Estado y aquellos estamentos que lo constituyen, entre ellos los políticos; la ciudadanía conformada por el resto. Los ciudadanos y las ciudadanas participan de la acción política, esporádicamente con su voto; al Estado, en ocasiones, se le identifica únicamente con los políticos.

Cuando los ciudadanos, debido a diversas motivaciones, utilizan los medios de comunicación o las movilizaciones sociales, reclamando la aplicación de leyes o la mejora de las mismas, por ejemplo, de temática ambiental o de salud, se configura el concepto de ciudadanía (Correa, 1998, p.86) y si estas personas participan en intervenciones públicas, manifestando intereses sociales, se puede hablar de participación ciudadana (Baño, 1998, p.36). La ciudadanía se incorpora así en la influencia del cuerpo político.

En el libro blanco de la Educación Ambiental se hizo énfasis en la necesidad de un nuevo impulso para la mejora y la conservación de nuestro entorno, y para alcanzarlo se indica entre otros aspectos el necesario fomento en "[...] la participación ciudadana en las iniciativas ambientales, como medio para alcanzar el consenso social que garantice el progreso hacia la sostenibilidad" (VV.AA., 1999, p.11). En esa línea Bourrut define la Educación Ambiental como un proceso de acción para el medio ambiente y fija como su objetivo "implicar a las personas en la resolución de los problemas ambientales" mediante participación activa. Por su parte Innerarity afirma que "la Educación Ambiental quiere promover la participación ciudadana para la resolución de la crisis que amenaza la salud del planeta”. Para este autor una vez hecha efectiva la democracia representativa, el voto, es preciso hacer efectiva la democracia participativa, para que la ciudadanía intente corregir el modelo clásico de la política que es incapaz de dar respuesta a los problemas ambientales (Bourrut e Innerarity, 2006).

Sin embargo, a pesar de estos planteamientos y asimismo teniendo presente que muchas de las acciones previstas en ellos se han puesto en marcha, tales como la implantación y desarrollo de infinidad de programas de Educación Ambiental, en empresas de todo tipo, comerciales, educativas, en la mayoría de las ocasiones prevalece el pensamiento económico y siempre, detrás de cualquier acción de relevancia, con importante inversión económica, aunque se levante la bandera ambientalista, hay que pensar en algún jugoso beneficio empresarial. Se echa en falta consenso 
social, la formación de los ciudadanos, la falta de argumentos que defiendan la conservación del medio a la par que la defensa de los puestos de trabajo y la generación de riqueza.

\section{DisCUSIÓN}

El espíritu de la LOGSE (Ley de Ordenación General del Sistema Educativo, 1990) de salpicar todo el curriculum con los contenidos transversales (Carratalá, 2016), se ha ido desdibujando poco a poco con las sucesivas normativas, dado que en éstas aparecen esos contenidos en nuevas asignaturas con un lugar concreto en el horario lectivo; pero otro de los motivos sin duda es una determinada manera de utilizar los libros de texto, convirtiendo esos contenidos relacionados con el comportamiento, en conceptuales, quedando los aspectos actitudinales nuevamente al albur de toda esa cantidad de variables a los que están sometidas las personas, sin referentes. Se podría afirmar que, en conjunto y en más casos de los deseables, el trabajo en valores se convierte en un aprendizaje conceptual de los mismos, que se ayuda a memorizar mediante determinadas actividades, concretas en el tiempo y con un valor ejemplificador que resulta poco eficiente.

Alcanzar la opinión acerca de cuál sería el comportamiento propio ante una situación determinada requiere discusión, reflexión y justificación y no es aceptable que la respuesta sea una mera repetición de lo que alguien nos dice respecto a lo que es lo mejor, lo que es bueno o es malo.

Existe poca información de la relación entre determinados comportamientos hacia el medio y la salud de las personas. En las aulas se incide en lo importante de reciclar materiales, pero no se indican cuáles son los riesgos del abuso en la utilización de esas materias más allá de su perjuicio al medio, sin vincularlo con la salud humana. Se puede considerar que se repiten mensajes conservacionistas para no dañar el medio natural, una información meramente ambiental, no relacionada con la salud humana, vinculación que no se relaciona de forma significativa, por lo que en los jóvenes puede prevalecer la idea de que un determinado comportamiento puede ser malo para el medio ambiente, pero no en qué medida puede afectar a su salud ni por qué motivo. Sería necesario valorar la conceptualización de los términos "salud" o "ambiente" por parte de los aprendices para poder evaluar la eficacia en los posibles comportamientos. El "saber" no condiciona un "comportamiento" determinado: es necesario el saber hacerlo y el querer realizarlo.

Habitualmente se habla de las energías y sus fuentes. Sin embargo, de forma generalizada cuando se tratan problemas ambientales derivados, tales como la desertización, el cambio de clima, o el calentamiento global, no se consideran otros aspectos fundamentales como las migraciones de pueblos debidas al agotamiento de recursos, a la radioactividad, al calentamiento global o a las guerras, en muchos casos consecuencia del comportamiento consumista de los habitantes de otras partes de la Tierra.

Si se considera que la salud ambiental es una meta formativa y que desde el sistema educativo reglado es preciso, y además posible, mejorar la situación actual respecto a los comportamientos de las personas, también habrá que considerar que no es suficiente con trabajar en el aula los contenidos estructurados en las materias o asignaturas tradicionales. Éstas, tienen habitualmente unos contenidos claramente delimitados y de forma mayoritaria es aceptado que pueden ser motivo de aprendizaje de forma individual y ocasionalmente mediante trabajos o realización de actividades grupales. Este planteamiento se puede generalizar a la mayoría de asignaturas que aportan una mayor cantidad de conocimientos de concepto y procedimiento al constructo denominado salud ambiental, como pueden ser los contenidos científicos relacionados con la sanidad, la geografía, las ciencias experimentales, las matemáticas, la historia, etc. Sin embargo, tenemos suficientes evidencias para considerar como un fracaso la falta de programación y desarrollo de las actividades que tienen una mayor carga de comportamiento social, aquellas que se no se sabe bien cómo se aprenden, pero se tienen indicios de su efectividad mediante la imitación, la discusión entre iguales, fuera del ámbito de una disciplina y con planteamiento de problemas que obliguen a la toma de decisiones, en pequeños grupos, por parte de los participantes es positiva para alcanzar las metas que se proponen. 
Sin duda estos planteamientos conducen al profesorado, obligado guía del aprendizaje del alumnado, a un mayor esfuerzo de preparación y adquisición de conocimientos, porque la actividad se desplaza alejándose de la denominada zona de confort, centrada en el docente, aquella en la que se siente más seguro con los materiales estructurados y contenidos delimitados, hacia una zona más incierta centrada en el aprendizaje del alumnado y, aún más, en su formación ciudadana solidaria.

A modo de conclusión final se puede apuntar que, si bien existe una agitación manifiesta en muchos ámbitos sociales a favor de los comportamientos ambientalistas, entre ellos en los centros docentes, la experiencia ha puesto de manifiesto que la mayoría de esos movimientos inciden en las personas, en el ser humano especie, como las beneficiarias de las acciones y en principio esto podría considerarse bueno, pero solo a corto plazo. Pero más aún estas acciones si son promovidas por estamentos oficiales, entiéndase Parlamento Europeo, países, comunidades autónomas, etc., al final, los aspectos económicos son los que hacen avanzar en un sentido determinado las acciones, siendo el medio el perjudicado. A modo de ejemplo, la etiqueta de sostenibilidad está evolucionando a la nueva, economía circular, cuando en realidad debiera denominarse economía helicoidal que provocará un aumento en el consumo debido a una mayor eficiencia en los procesos, y un mayor gasto energético debido a los procesos intermedios de recuperación, siendo a la postre negativo para el medio, pero sin duda con una mayor generación de empleo y dinamización económica. Por otra parte, se puede provocar un retroceso en aspectos directos de riesgos para la salud de las personas, como por ejemplo en la comercialización de leche cruda, olvidando todos avances de la ciencia en sanidad animal. Un ejemplo paradigmático lo encontramos en la fabricación de vidrio utilizado como ejemplo de sostenibilidad, de reciclado y ahora de economía circular. El aumento en la fabricación de envases de vidrio y del consumo de sus productos ha sido espectacular en las últimas décadas; sin embargo, lo que se publicita es que ha aumentado el reciclado. Sin duda la salud de las personas es fundamental y prioritario, pero la salud del medio será el condicionante de la primera.

La formación de los ciudadanos se fundamenta en la educación en los centros docentes y una herramienta fundamental son las metodologías de debate, de argumentación, dirigidas por profesorado bien formado, no solamente en el entrenamiento del debate, sino en conocimientos acerca de los problemas ambientales reales y cómo éstos repercuten en la salud de las personas. Son necesarios los argumentos científicos y ser capaz de utilizarlos. Son los agentes políticos y económicos los que lideran las acciones importantes a favor del medio, pero en el fondo sus intereses son otros. Sus preguntas y estudios responden a preguntas similares a: ¿Repercute la etiqueta ecológica en la venta de productos?, ¿Cómo repercute en las ventas la eliminación del aceite de palma en los alimentos de mis estantes? Y mientras el consumismo helicoidal sigue avanzando.

\section{Referencias}

Álvaro, N., Mayoral, O. y Gavidia, V. (2017). Competencias en salud ambiental de los jóvenes valencianos. Enseñanza de las Ciencias, número extraordinario, 811-816.

Arasa, H., Argudo, J., Ayuso, A., Carrasquer, J., Escario, M. J., Espinosa, M. L., Faustino, D., Franco, J. M., Gallego, J., Granizo, C., Muñoz, M., Plumed, M. y Tremps, M. (1991). Educación para la Salud: propuestas para su integración en la escuela. Zaragoza: Ministerio de Educación y Ciencia, Diputación General de Aragón, Departamento de Sanidad, Bienestar Social y Trabajo.

Baño, R. (1998). Participación Ciudadana: Elementos Conceptuales. En Correa, E. y Noé, M. (Eds.), Nociones de una ciudadanía que crece, 15-37. Santiago de Chile: FLACSO Chile.

Barlow, J. P. (1996). Declaración de Independencia del Ciberespacio. Recuperado de http://www.uhu.es/ramon.correa/nn_tt_edusocial/documentos/docs/declaracion_independenci a.pdf [23 de abril de 2018]. 
Bourrut, H. y Inneraritu, D. (2006). Educación Ambiental, participación ciudadana, desarrollo sostenible y Agenda 21 local. III Jornadas de Educación Ambiental de la Comunidad Autónoma de Aragón, 24, 25 y 26 de marzo. Zaragoza: Gobierno de Aragón.

Bula, G. (2010). Cinco habilidades para el siglo XXI. Revista Sustentabilidades, 1(1), 1-15.

Bula, G. (2011). Educación Ambiental: Del enfoque por competencias al enfoque por habilidades. Magistro, 5(10) 33-42. $\quad$ Recuperado de https://dialnet.unirioja.es/servlet/articulo?codigo=3945764 [10 de junio de 2019].

Carratalá, A. (2016). Reflexiones sobre la transversalidad. En V. Gavidia (Coord.) Los Ocho Ámbitos de la Educación para la Salud en la Escuela (pp. 19-42). Valencia: Tirant Humanidades.

Casado, D. (2008). Coordinación (gruesa y fina) en y entre los servicios sanitarios y sociales. Barcelona: Hacer Editorial.

Castorina, J. A., Barreiro, A. y Toscano, A. G. (2005). Las representaciones sociales y las teorías implícitas: una comparación crítica. Educação \& Realidade, 30(1), 201-222.

Comisión de las Comunidades Europeas (2003). Comunicación de la Comisión al Consejo, al Parlamento Europeo y al Comité Económico y Social Europeo: Estrategia Europea del Medio Ambiente y salud. Recuperado de https://eurlex.europa.eu/LexUriServ/LexUriServ.do?uri=COM:2003:0338:FIN:ES:PDF [10 de junio de 2019]

Conselleria de Educación, Investigación, Cultura y Deporte (2017). Resolución conjunta de 11 de diciembre de 2017, de la Conselleria de Educación, Investigación, Cultura y Deporte y de la Conselleria de Sanidad Universal y Salud Pública, por la que se dictan instrucciones para la detección y la atención precoz del alumnado que pueda presentar un problema de salud mental. [2017/11874]. Diari Oficial de la Generalitat Valenciana, núm 8196, del 22 de diciembre de 2017, 48164-48185. Recuperado de https://www.dogv.gva.es/datos/2017/12/22/pdf/2017_11874.pdf [10 de junio de 2019].

Correa, E. (1998). Participación ciudadana y gobernabilidad. En Correa, E. y Noé, M. (Eds.), Nociones de una ciudadanía que crece (pp.83-89). Santiago de Chile: FLACSO Chile.

De Marcos, A. (2011). El fin de una larga travesía: La ley 41/2010 de Protección del Medio Marino. Ambienta, 94. Recuperado de:

http://www.revistaambienta.es/WebAmbienta/marm/Dinamicas/secciones/articulos/Anamarcos.htm [10 de junio de 2019].

Dreyfus, S. E. y Dreyfus, H. L. (1980). A Five-Stage Model of the Mental Activities Involved in Directed Skill Acquisition. Washington, DC: Storming Media.

Dreyfus, H. L. y Dreyfus, S. E. (2003). "The Challenge of Merlau-Ponty's Phenomenology of Embodiment for Cognitive Science”. En Weiss, G. y Fern Haber, H. (Eds.), Perspectives on Embodimentpp (pp. 103-120). Londres: Routledge.

Dreyfus, S. E. (2004). The Five-Stage Modelo f Adult Skill Acquisition. Bulletin of Science, Technology \& Society, 24(3), 177-181. DOI: 10.1177/0270467604264992

Fabre, J. H. (1882). Nouveaux Souvenirs Entomologiques, vol. II. París: Delagrave.

Fernández Enguita, M. (2016). La Educación en la Encrucijada. Madrid: Fundación Santillana.

Furió, C. y Gil Pérez, D. (1978). El programa-guía: una propuesta para la renovación de la didáctica de la Física y Química en el Bachillerato. Barcelona: Instituto de Ciencias de la Educación de la Universidad de Barcelona.

García Díaz, J. E. (2003). Investigando el ecosistema. Investigación en la Escuela (monográfico: Proyecto curricular Investigando Nuestro Mundo), 51, 83-100.

García Díaz, J. E. (2004). Los contenidos de la Educación Ambiental: una reflexión desde la perspectiva de la complejidad. Investigación en la Escuela (monográfico: Complejidad y educación), 53, 31-52. 
Gavidia, V. (2016). El Proyecto Comsal (Competencias en Salud). En V. Gavidia (Coord.), Los Ocho Ámbitos de la Educación para la Salud en la Escuela (pp. 3-18). Valencia: Tirant Humanidades.

González de Haro, M.D. (2008). La educación para la salud: “asignatura pendiente” para la escuela. XXI Revista de Educación, 10, 123-136. Recuperado de: http://www.uhu.es/publicaciones/ojs/index.php/xxi/article/viewFile/506/733 [10 de junio de 2019].

Harlen, W. (2010). Principios y grandes ideas de la educación en ciencias. Edición en castellano por Rosa Devés. Recuperado de http:// www.innovec.org.mx [10 de junio de 2019].

Hervás de la Torre, A. (2011). La participación comunitaria en Salud y el Trabajo Social Sanitario. Documentos de Trabajo Social, 50, 146-186.

Ley Orgánica 1/1990, de 3 de octubre de 1990, de Ordenación General del Sistema Educativo. Boletín Oficial del Estado, núm. 238, de 4 de octubre de 1990, págs. 28927 a 28942. Recuperado de https://www.boe.es/boe/dias/1990/10/04/pdfs/A28927-28942.pdf [10 de junio de 2019].

Marchioni, M. (2004). La acción social en y con la comunidad. Zaragoza: Libros Certeza.

Meira, P. A. (2013). Problemas ambientales globales y educación ambiental. Integra Educativa, VI(3), 29-64.

Maeterlinck, M. (1967). La vida de los termes (Comejenes u hormigas blancas). Madrid: Espasa Calpe.

Marina, J. A. (2012). La inteligencia ejecutiva. Barcelona: Editorial Planeta, Ariel.

Ministerio de Educación, Cultura y Deporte (2017). Datos y Cifras, curso escolar 2017/18. Madrid: Secretaría General Técnica.

Naess, A. (1973). The Shallow and the Deep, Long-Range, Ecology Movement. A Summary. Inquiry, An Interdisciplinary Journal of Philosophy, 16(1-4) 95-100.

OECD (2017). Education at a Glance 2017: OECD Indicators. Paris: OECD Publishing. http://dx.doi.org/10.1787/eag-2017-en

Pareja, J. A. (2011). Modelos globalizadores y técnicas didácticas interdisciplinares. En Lorenzo, M. (Coord.), Didáctica para la educación infantil, primaria y secundaria (pp. 167-198). Madrid: Universitas.

Ponz, A., Carrasquer, B., Álvarez, M. V. y Carrasquer, J. (2017). Percepción de las competencias en salud de los libros de texto por estudiantes de magisterio y expertos. Enseñanza de las Ciencias, número extraordinario, 817-822.

Pueyo, A., Carrasquer, J., Álvarez, M. V. y Ponz, A. (2016). El Centro de Salud como Escuela para la Salud. En Fontenelle, A. M., Cesar Praça, C. y de Araújo Oliveira, D. (Coord.), Avaliação de Processos Educacionais em Saúde: Tendências e Inovações (pp. 220-244). Fortaleza: Editora da Universidade Estadual do Ceará.

Rodrigo, M. J. (1994). Etapas, contextos, Dominios y Teorías Implícitas en el Conocimiento Social. En Rodrigo, M. J. (Ed.), Contexto y Desarrollo Social (pp. 21-43). Madrid: Síntesis Psicología.

Sotolongo, P. L. y Delgado, C. J. (2006). La revolución contemporánea del saber y la complejidad social. Hacia unas ciencias sociales de nuevo tipo. Buenos Aires: Consejo Latinoamericano de Ciencias Sociales.

Talavera, M. y Gavidia, V. (2013). Percepción de la Educación para la Salud en el personal docente y sanitario. Didáctica de las Ciencias Experimentales y Sociales, 27, 115-129.

Tobón, S. (2007). El enfoque complejo de las competencias y el diseño curricular por ciclos propedéuticos. Acción Pedagógica, 16, 14-28.

Vélaz de Medrano, C., Manzano, N. y Blanco, A. (2013). Los procesos de cambio de las políticas públicas sobre orientación y apoyo a la escuela: análisis comparado de sistemas vigentes y 
emergentes. Estudio múltiple de casos en una muestra de comunidades autónomas. Madrid: Ministerio de Educación, Cultura y Deporte.

VV.AA. (1999). Libro Blanco de la Educación Ambiental en España. Madrid: Ministerio de Medio Ambiente. Recuperado de http://www.oei.es/historico/decada/portadas/libroblanco.htm [10 de junio de 2019].

Zabala, A. (1999). Enfoque globalizador y pensamiento complejo. Una respuesta para la comprensión e intervención en la realidad. Barcelona: Graó.

\section{Cómo CITAR ESTE ARTÍ́CULO}

Carrasquer, J., Ponz, A., Carrasquer, B. y Álvarez, M. V. (2019). Reflexiones acerca de la salud ambiental. Didáctica de las ciencias experimentales y sociales, 36, 115-128. DOI: 10.7203/DCES.36.12990. 
